\title{
MOSAIC ARTHROPLASTY OF THE MEDIAL FEMORAL CONDYLE IN HORSES - AN EXPERIMENTAL STUDY ${ }^{\#}$
}

\author{
Gábor Bodó ${ }^{1,2^{*}}$, Gábor VÁSÁRHELYI ${ }^{3}$, László HANGODY ${ }^{3}$ and László MóDIS ${ }^{4}$ \\ ${ }^{1}$ Large Animal Clinic, Faculty of Veterinary Science, Szent István University, \\ Üllő, Hungary; ${ }^{2}$ ISME, Equine Hospital, Vetsuisse Faculty, University of Bern, \\ Switzerland; ${ }^{3}$ Uzsoki Hospital, Orthopaedics and Trauma Department, Budapest, \\ Hungary; ${ }^{4}$ Department of Anatomy, Histology and Embryology, Faculty of Medicine, \\ Debrecen, Hungary
}

(Received 7 November 2012; accepted 2 July 2013)

One Arabian and 5 Hungarian half-bred horses were used to study the macroscopic and microscopic survival of autologous osteochondral grafts in the weight-bearing surface of the medial femoral condyle (MFC). Grafts were harvested from the cranial surface of the medial femoral trochlea (MFT) under arthroscopic control. Three of them were transplanted into the weight-bearing surface of the contralateral MFC using an arthrotomy approach. Three months later this transplantation procedure was repeated on the opposite stifle joints in the same animals, but at that time transplantation was performed arthroscopically. Follow-up arthroscopy was carried out 12 months after the first operations, and biopsies were taken from both the recipient and the donor sites for histological examination. During follow-up arthroscopy, the transplanted areas looked congruent and smooth. Microscopically, the characteristics of hyaline cartilage were present in 5 out of the 10 biopsies examined; however, in the other half of biopsies glycosaminoglycan (GAG) loss and change in the architecture of the transplanted cartilage was observed. In a 16-year-old horse, all grafts broke during harvesting, and thus transplantation was not performed. No radiological signs of osteoarthritic changes were detected 9 to 12 months after the operations in the donor and recipient joints. Clinically, no lameness or effusion was present three months after the transplantations.

Key words: Mosaicplasty, stifle, joint, horse, resurfacing, cartilage

In both human and equine patients, focal cartilaginous lesions can result from chondropathy or chondral trauma and are difficult to treat, especially on weight-bearing joint surfaces (Hangody et al., 2010; Nixon et al., 2011). In terms

${ }^{\#}$ This work was performed at the Large Animal Clinic, Faculty of Veterinary Science, Szent István University, Üllő, Hungary

"Corresponding author; Present address: ISME, Equine Hospital, Vetsuisse Faculty, University of Bern, Länggass-Strasse 124, 3012 Bern, Switzerland;

E-mail: gabor.bodo@vetsuisse.unibe.ch 
of its biology and healing potential, the equine articular cartilage is similar to the human one, but completely different from that of laboratory animals such as the rabbit, where cartilage healing is exuberant and often complete (Hurtig et al., 2001). Following a full-thickness lesion of hyaline cartilage, there is no regeneration but a reparation process is possible, which results in fibrocartilage with biomechanical properties inferior to those of hyaline cartilage. The size, location and age of the defect are further factors determining the quality of repair tissue after such a joint cartilage injury. The resulting fibrocartilaginous repair tissue can undergo fibrillation and degeneration (Martinek et al., 2003).

Several techniques have been tested in an attempt to achieve a functional weight-bearing joint surface in equine patients. Cancellous bone grafting of the medial femoral condyle (MFC) was characterised by the presence of dead graft and secondary cyst formation compared to ungrafted defects which were filled with fibrous tissue without identifiable cyst formation (Jackson et al., 2000).

Perichondrial grafts do not have the collagen arcades and dense collagenous superficial layer to withstand the biomechanical forces that occur during weight bearing. Therefore, they are not suited for reconstruction of the weightbearing joint surface (Hurtig et al., 2001). In a recent equine study, a 74\% success rate was reported when treating subchondral bone cysts of the MFC with cancellous bone grafts covered by growth-factor-enhanced chondrocytes (Ortved et al., 2012). However, histological examination of the resulting surface was not performed in that clinical case series.

In an equine experimental study, $1-\mathrm{cm}^{2}$ full-thickness chondral defects were created on both MFCs; one side was treated with microfracture, while the other was left untreated. Histomorphometry confirmed more repair tissue and an increased percentage of type II collagen filling microfracture-treated defects as compared to untreated defects (Frisbie et al., 1999). However, none of the abovementioned methods resulted in a repair tissue with hyaline or hyaline-like cartilage quality.

Osteochondral allograft (Pearce et al., 2003) and autograft (Hurtig et al., 2001) studies in the equine fetlock and carpal joints, respectively, resulted in a superior gliding surface with macroscopic and microscopic features of hyaline cartilage compared to the above-described techniques; however, glycosaminoglycan (GAG) concentration was decreased in the grafts.

Evaluating the results of knee autologous osteochondral mosaicplasty (MP) with 303 elite human athletes over a 9.6-year period, good to excellent clinical results were observed in $91 \%$ of those athletes. Based on clinical experience in over 1000 MP patients, this technique has become a proven useful alternative for the treatment of 1.0- to 4- $\mathrm{cm}^{2}$ focal chondral and osteochondral lesions in competitive human athletes (Hangody et al., 2008; Hangody et al., 2010). Clinical experience in the stifle (Bodó et al., 2000; Bodó et al., 2004) and hock (Janicek et al., 2010) joint of horses showed a favourable clinical outcome in all cases. 
The results of MP in the MFC are well described in different animal models (dog: Hangody et al., 1997; sheep: Huang et al., 2004; pig: Harman et al., 2006); however, to the authors' knowledge no information on the histological appearance of transplanted areas and donor sites has been reported in the horse stifle.

The objective of this study was to present the macroscopic and microscopic results of donor and recipient areas after MP surgery carried out in the equine stifle.

\section{Materials and methods}

\section{Surgical procedure}

Six horses aged 8 months to 16 years (mean age: 5.8 years) were included in this study. The distribution of the horses according to gender and breed is presented in Table 1 . The horses were screened preoperatively using radiographs of the stifle and systematic lameness evaluation. In full compliance with the Act on Animal Experiments, this study was evaluated and approved by the Ethics Committee of Szent István University, Hungary. Inclusion criteria were no radiographic findings of the stifle and absence of lameness during clinical examination including negative flexion tests.

Antibiotics $(4,000 \mathrm{U} / \mathrm{kg}$ benzathine penicillin, 1,000 U/kg procaine penicillin and $6.2 \mathrm{mg} / \mathrm{kg}$ dihydrostreptomycin sulphate, Tardomyocel comp. III i.m., Bayer Hungaria Ltd.) and $3.0 \mathrm{mg} / \mathrm{kg}$ phenylbutazone i.v. were given prior to surgery.

After induction of general anaesthesia, the horses were positioned in dorsal recumbency with the donor limb maximally extended and the recipient limb in flexed position (Bodó et al., 2000; Bodó et al., 2004). The cranial aspect of the medial femoral trochlea (MFT) of a randomly selected right or left limb was used as a donor site. Four to five cylindrical osteochondral grafts $(6.5 \mathrm{~mm}$ in diameter, 35-40 mm long, spaced approximately $5 \mathrm{~mm}$ from each other) were harvested under arthroscopic guidance using a conventional arthroscopic approach. The steps of the harvesting procedure and the instruments used were the same as described previously (Bodó et al., 2004). In the 16-year-old horse, all harvested grafts fractured $2-5 \mathrm{~mm}$ under the hyaline cap of both limbs, so transplantation was not performed (Fig. 1). This horse was excluded from follow-up evaluations.

Arthrotomy (with a 50- to 60-mm-long skin incision) of the contralateral medial femorotibial joint was carried out under the same general anaesthesia to implant the grafts. Three grafts, $30 \mathrm{~mm}$ in length, were transplanted into the MFC in a craniocaudal line, leaving an approximately $2-\mathrm{mm}$ space between the grafts. The recipient hole was drilled with a $6.5-\mathrm{mm}$ drill bit $30 \mathrm{~mm}$ in depth and flushed with isotonic saline. The drilled hole was finally dilated with a calibrated conical dilator $30 \mathrm{~mm}$ in depth. Grafts were delivered in a press-fit fashion through the drill guide with the help of an adjustable tamp and seated as flush as possible with the surrounding cartilage (Bodó et al., 2004). The location of each 
donor and recipient area was recorded on diagrams of the joint surface (Fig. 2). Closure of the surgical wound was performed in five layers.

\section{Table 1}

Data of horses included in the study, with special regard to histological results of the recipient sites 9 to 12 months after transplantation

\begin{tabular}{|c|c|c|c|c|c|c|c|c|}
\hline \multirow{2}{*}{$\begin{array}{l}\text { Horse } \\
\text { No. }\end{array}$} & \multirow{2}{*}{$\operatorname{Age}^{* *}$} & \multirow[b]{2}{*}{ Sex } & \multirow[b]{2}{*}{ Breed } & \multicolumn{5}{|c|}{ Histological data - implanted grafts } \\
\hline & & & & $\begin{array}{c}\text { Graft age, } \\
\text { months }\end{array}$ & Interface $^{\dagger}$ & Surface $^{\dagger \dagger}$ & Thickness & Quality \\
\hline $1 \mathrm{~A}$ & $8 \mathrm{~m}$ & gelding & Arabian & 12 & 1 & 1 & 1 & 1 \\
\hline $1 \mathrm{~B}$ & $8 \mathrm{~m}$ & gelding & Arabian & 9 & 2 & 1 & 1 & 1 \\
\hline $2 \mathrm{~A}$ & 6 & gelding & $\mathrm{HHB}^{* * *}$ & 13 & 2 & 1 & 2 & 4 \\
\hline $2 B$ & 6 & gelding & $\mathrm{HHB}^{* * *}$ & 10 & 3 & 2 & 1 & 2 \\
\hline 3B & 4 & gelding & НHB & 12 & 2 & 1 & 1 & 1 \\
\hline $3 \mathrm{~A}$ & 4 & gelding & ННВ & 9 & 4 & 1 & 1 & 1 \\
\hline $4 B$ & 3 & mare & ННВ & 12 & 2 & 1 & 2 & 2 \\
\hline $4 \mathrm{~A}$ & 3 & mare & НHB & 9 & 2 & 2 & 2 & 2 \\
\hline $5 \mathrm{~A}$ & 5 & mare & НHB & 13 & 1 & 1 & 2 & 1 \\
\hline $5 B$ & 5 & mare & НHB & 10 & 3 & 1 & 3 & 4 \\
\hline $6 \mathrm{~B}$ & 16 & gelding & ННВ & - & - & - & - & - \\
\hline $6 \mathrm{~A}$ & 16 & gelding & НHB & - & - & - & - & - \\
\hline
\end{tabular}

${ }^{*} \mathrm{~A}=$ left hind limb, $\mathrm{B}=$ right hind limb; ${ }^{* *}$ At the time of transplantation (in years). $8 \mathrm{~m}=$ eight months; ${ }^{* * *}$ Hungarian Half-bred; ${ }^{\dagger} 1=$ no gap; $2=$ minor fissure $(<10 \%$ of cartilage thickness $) ; 3=$ gap $(=50 \%$ of cartilage thickness); $4=$ full-thickness gap between host and transplanted cartilage; ${ }^{\dagger}$ Surface of transplanted cartilage; $1=$ smooth without fissures; $2=$ minor fissures; 3 = more fissures on the cartilage; $4=$ degenerative changes with cartilage fibrillation; ${ }^{\sharp} 1=$ transplanted cartilage thickness is between $75-100 \%$ of surrounding cartilage thickness; $2=50-75 \%, 3=25-50 \%$, $4=<25 \%$; ${ }^{*}$ Type of transplanted cartilage: $1=100 \%$ hyaline cartilage (see Fig. 7A, B, C); $2=$ predominance of hyaline cartilage (more than $60 \%$ hyaline cartilage, see Fig. $8 \mathrm{~A}, \mathrm{~B}, \mathrm{C}$ ); $3=$ predominance of fibrocartilage (more than $40 \%$ fibrocartilage); 4 = fibrocartilage $(100 \%$ fibrocartilage, see Fig. 8D)

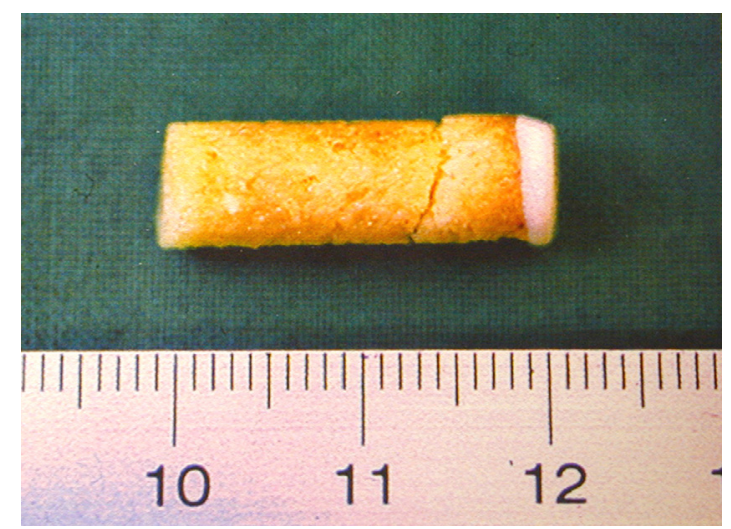

Fig. 1. Fractured subchondral region of a harvested graft $6.5 \mathrm{~mm}$ in diameter in the 16-year-old horse 


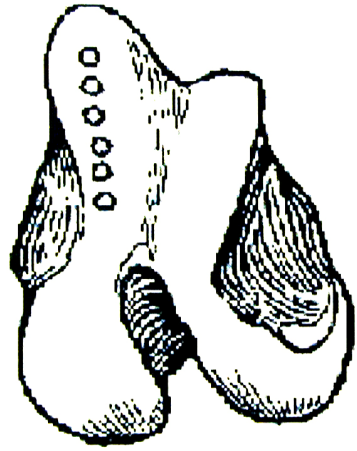

Right dist. femur

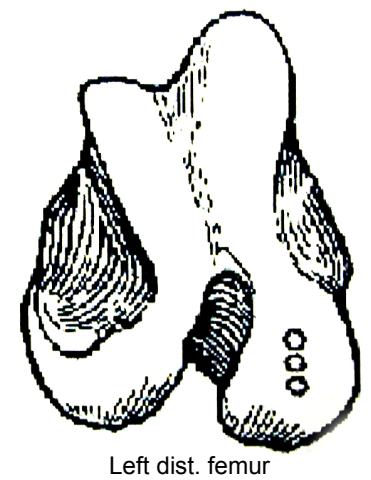

Left dist. femur

Fig. 2. Schematic drawing of the donor and recipient sites

Three months after the first operation the same transplantation procedure was performed on the opposite stifles. The only difference was that grafts were inserted under arthroscopic control, using a lateral arthroscopic approach and choosing the dorsal instrument portal for insertion of the grafts.

\section{Clinical examination and $X$-rays}

Lameness was evaluated by the same observer (GB) at the walk during the first week, and once a week during the first three months. None of the horses showed lameness three months after the surgeries at the walk or trot. In the last six months of the study, the horses were evaluated once every month. Upper hindlimb flexion tests were performed in each horse 3 to 6 months after the first surgery. Lameness scores from 0 to 5 were used according to the American Association of Equine Practitioners (AAEP) scoring system. Clinical evaluation of the stifle joints for effusion and swelling was also documented before each lameness examination. Radiological examination ( $\mathrm{CaL}-\mathrm{CrM}, \mathrm{CaCrO})$ was carried out 12 months after the first transplantations.

\section{Postoperative care}

One-week box rest followed by a two-week hand walk was recommended for the horses. After three weeks of controlled exercise, the horses were turned out in a small paddock. Oral phenylbutazone ( $2 \mathrm{mg} / \mathrm{kg}$ once daily) was administered for 4-10 days postoperatively after all operations.

\section{Sample collection and arthroscopic evaluation}

This study was not a terminal experiment. For evaluating our results, all horses underwent an arthroscopic examination of the donor as well as the transplanted areas. This examination was performed at the time of harvesting the osteochondral biopsies, 12 months after the first transplantations. 
During arthroscopy the grafts and donor fields were evaluated for discoloration, loss of cartilage, fissure development, firmness and congruency, and the joints for any signs of osteoarthritis. Osteochondral biopsies $6.5 \mathrm{~mm}$ in diameter were taken with a tubular chisel (Metrimed Ltd., Hódmezővásárhely, Hungary) under arthroscopic control at the border between the host and the transplanted cartilage (one biopsy per joint) as well as at the border between donor canals and surrounding cartilage for histological examination.

\section{Histological evaluation}

Following routine preparation, every third section was stained with haematoxylin and eosin (HE), picrosirius red (PSR) (Constantine and Mowry, 1968) and dimethylmethylene blue (DMMB) (Módis, 1991). Histological sections were assessed for quality of the transplanted hyaline cartilage (scores 1-4), graft articular cartilage thickness compared with the surrounding cartilage, interface between host bed and transplanted cartilage, and fissuring of the surface (scores 1-4). Further details are presented in Table 1. The histological sections were also examined at the subchondral area for bony union and presence of any abnormalities (collapse of the graft, cyst formation, etc.).

\section{Results}

\section{Clinical and radiographic examination}

Moderate femoropatellar joint effusion of the donor extremities persisted during the first 3-4 weeks after surgery. All horses showed a stiff gait on the donor site during that 3- to 4-week period, which disappeared together with the joint effusion within two months after the operations. Less effusion but $1-2 / 5$ degree of lameness was detected on the recipient extremities during the first two weeks. In the second week, two horses were severely lame (3-4/5) in the recipient limb, which improved over 10 to 14 days. These horses received NSAIDs for an extended period (phenylbutazone orally in a dose of $4 \mathrm{mg} / \mathrm{kg}$ for 5-7 days followed by $2 \mathrm{mg} / \mathrm{kg}$ for further 10 days).

Closure of one recipient site was repeated on the second postoperative day under short general anaesthesia in one case because of wound dehiscence during recovery. No wound healing complications were noted in the donor or recipient wounds. Lameness was not apparent in any animal two months after the first or the second operation. The proximal flexion test was negative on both hind legs six months after the first operations (three months after the second operations) except for one case, where grade 3/5 lameness was observed on a $10-15 \mathrm{~m}$ distance after a flexion test at one site (during the second operation it was a recipient joint). No significant pathological bony changes were seen in any of the stifle joints during radiological evaluation, with the exception of some unevenly out- 
lined subchondral bone plate in 4 out of 10 MFCs. On the MFT, some of the donor canals still appeared slightly less radiopaque than the surrounding bone without any signs of osteoarthritis.

\section{Follow-up arthroscopy}

No osteoarthritic changes were visible macroscopically in the donor joints. A smooth gliding surface covered most of the donor canals. In 3/60 donor canals mild fibrillation of the surface was detected (Fig. 3A, B).

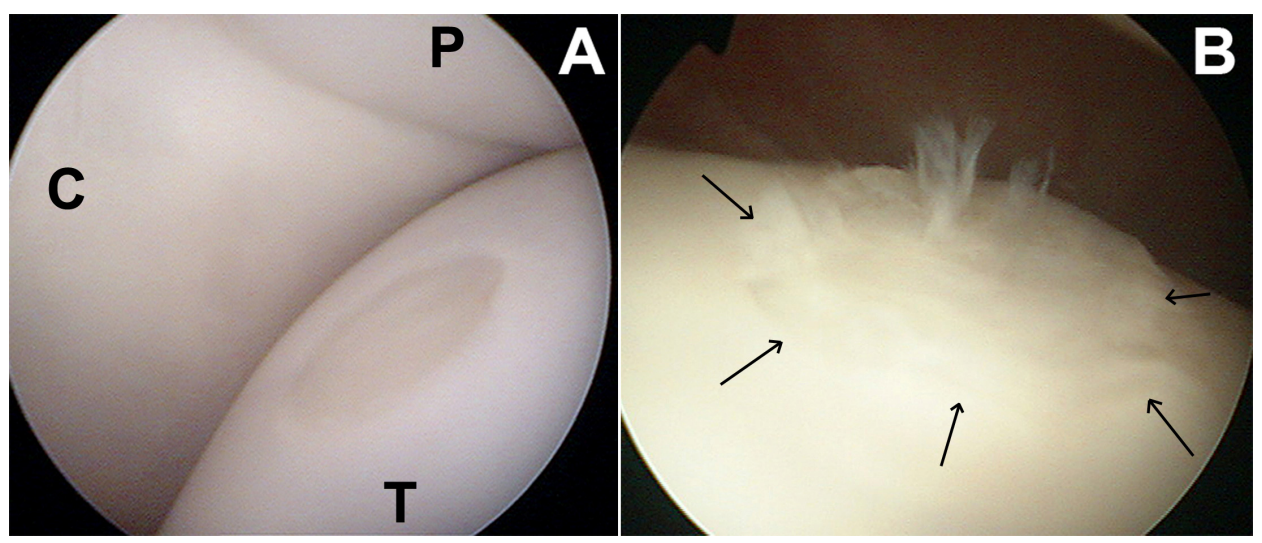

Fig. 3. Follow-up arthroscopic images of the donor sites (medial femoral trochlea).

Figure 3A: a smooth and congruent even surface covering the donor canal 12 months after harvesting. P: patella, T: medial femoral trochlea, C: parapatellar cartilage. Figure 3B: fibrillation of the surface on a previous donor canal 12 months following harvest (the outline of the previous donor canal is indicated by arrows)

No gross degenerative changes were detected in the medial femorotibial joints during arthroscopic evaluation. A smooth surface without any abnormalities was observed in 12/30 transplanted grafts (3/15 with arthroscopic and 9/15 with arthrotomic insertion). Further information about arthroscopic evaluation is presented in Table 2 and Fig. 4A, B, C. In general, more favourable macroscopic results were achieved through arthrotomic insertion.

\section{Table 2}

Macroscopic appearance of the transplanted grafts during follow-up arthroscopy

\begin{tabular}{lcccccc}
\hline Type of insertion & $\begin{array}{c}\text { Smooth surface } \\
\text { without }\end{array}$ & $\begin{array}{c}\text { Too deep } \\
\text { innormalities }\end{array}$ & insertion & & $\Sigma$ & \multicolumn{3}{c}{ Non-perpendicular insertion } \\
\cline { 4 - 6 } with fissures & $\begin{array}{c}\text { Irregularly } \\
\text { ripping surface }\end{array}$ \\
\hline Arthrotomic (15) & 9 & 2 & 4 & 3 & 1 \\
Arthroscopic (15) & 3 & 1 & 7 & 3 & 4 \\
\hline
\end{tabular}




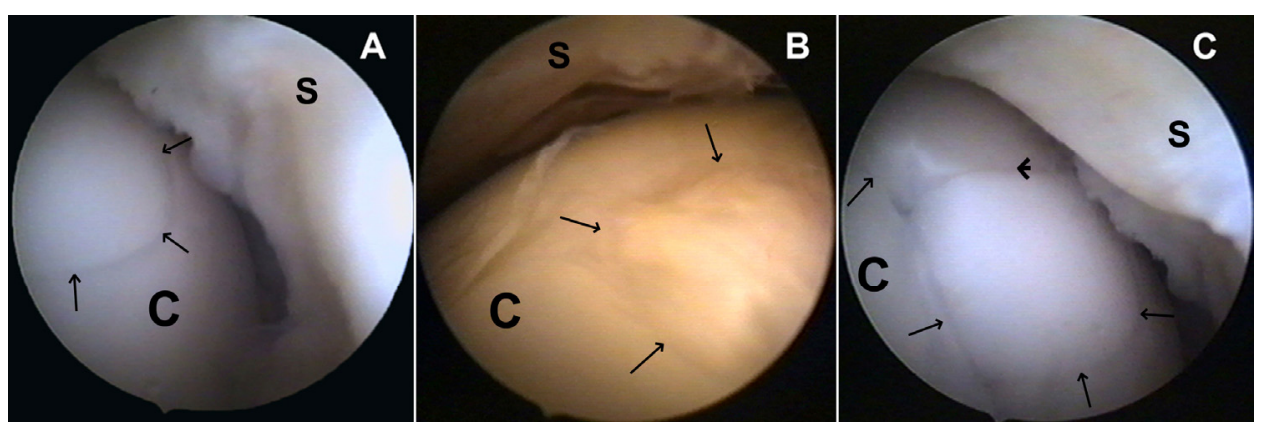

Fig. 4. Follow-up arthroscopic images of the recipient sites. Figure 4A: interfaces between the host bed and transplant are hard to recognise after 12 months. Figure 4B: a congruent but irregularly rippling surface of a transplant. Figure 4C: protuberance of the transplanted graft cap and a small gap formation at the interface region between host bed and transplant. S: synovial membrane,

C: medial femoral condyle; arrows indicate the interface between transplanted graft and surrounding cartilage; arrowhead: interface between two transplanted grafts

\section{Histological examination}

In half of the samples $(5 / 10)$ taken from the host-graft border areas, all the characteristics of normal articular cartilage could be observed both in graft and host regions (Fig. 5A, B, C), while in 3/10 samples a predominance of hyaline cartilage was visible (Fig. 6A, B, C). A union between the transplanted and the host hyaline cartilage could be observed in those samples (Figs 5 and 6). All the characteristics of a living transplanted hyaline cartilage were shown by the properly stained nuclei of the chondrocytes and the amount of GAG in the extracellular matrix (ECM) indicated by the homogeneously intense staining with DMMB (Fig. 6A, B, C and Fig. 8A, B). Both collagen fibrils and GAG molecules exhibited optical anisotropy in the ECM of the superficial and deep zones as revealed by polarised light microscopic observations (Fig. 5B, C). In 2/10 cases the transplanted articular cartilage was partially transformed into fibrocartilage (Fig. 6A, $\mathrm{B}, \mathrm{C})$. In one case, a cartilage/bone cylinder was inadvertently harvested from the craniomedial part of the medial femoral trochlea, where the hyaline cartilage was significantly thinner than that of the cranial surface of the MFC, used in all other implantations.

Full bony union was observed in all recipient area samples at the subchondral bone level. No cyst formation, advanced bony sclerosis, necrosis or lysis was detected at the subchondral level in any of the evaluated samples.

The donor channels were rebuilt with cancellous bone and covered by fibrocartilage (Fig. 7B, C). In a 20-month-old gelding mainly hyaline cartilage covered the examined donor channel (Fig. 7A). The histological data are summarised in Table 1. 


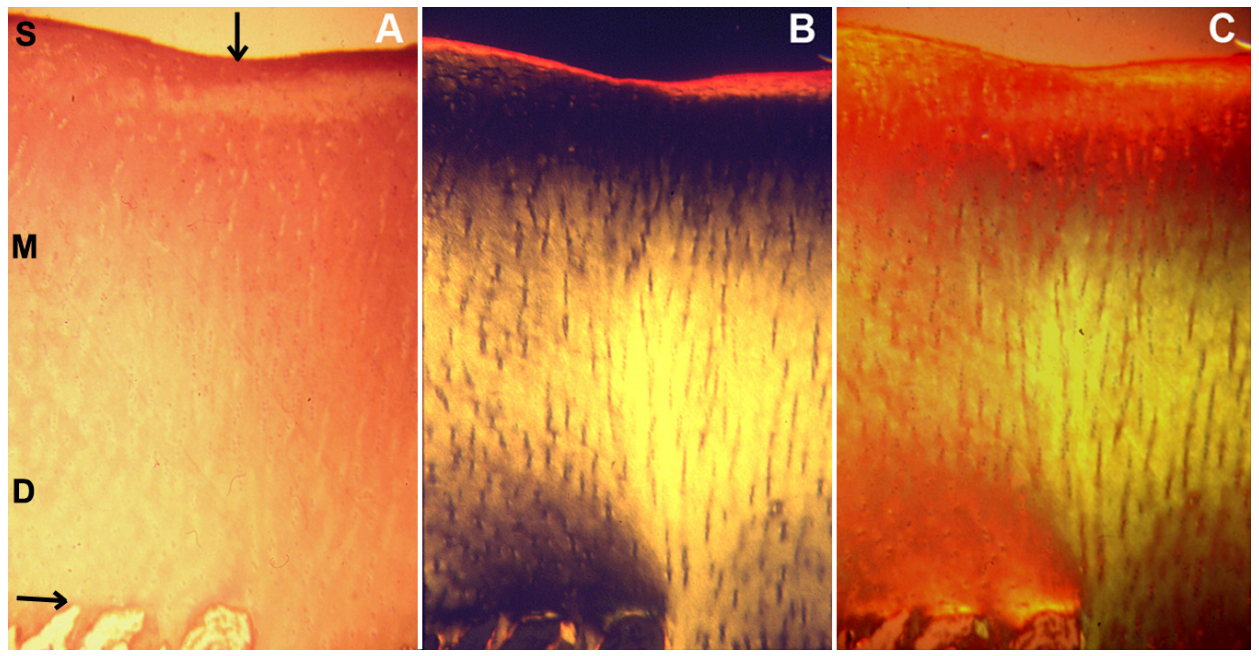

Fig. 5A, B, C. Articular cartilage section showing the host-graft interface region 9 months after autologous osteochondral mosaicplasty (MP) with picrosirius red staining for collagen taken from the same visual field in normal (A) and polarised light microscope (B, C) without (B) and after (C) compensation of the optical anisotropy. The host-graft border is shown by minor articular surface concavity (arrow). The collagen structure of superficial zones (S) is strongly stained and birefringent, while a moderate staining and optical anisotropy is seen in the deep zones (D) both in host and graft areas (A, B). The intermediate zone (M) is isotropic (dark) in polarised light microscope

$(B, C)$. After compensation of the optical anisotropy with a $\lambda / 4$ compensator plate, different polarisation colours appear in the extracellular matrix of the superficial and deep zones (orange and bluish green, respectively), suggesting the different orientation pattern of collagen fibrils in these zones (tangential orientation in the superficial zone and radial orientation in the deep zone). All of these optical characteristics are typical of the normal articular cartilage.

Microscopic magnifications: $\times 20$

\section{Discussion}

Transplantation of autologous osteochondral grafts into the MFC resulted in full survival of the transplanted hyaline cartilage in $50 \%$ of the biopsies examined 9 to 12 months following insertion, whereas in the other half of the examined biopsies, hyaline-like cartilage or full transformation to fibrocartilage was observed. A bony union between the graft and the surrounding subchondral bone was achieved in all cases. No signs of osteoarthritis were observed 9-12 months after transplantation in the recipient (medial femorotibial) and donor (femoropatellar) joints. In an equine study of the MFC it was stated that the articular cartilage can show irreversible degenerative changes already three months after initiated cartilage injury (Bolam et al., 2006). In this study, the horses were evaluated after 12 months, which allowed us to detect advanced cartilage degeneration if present. 


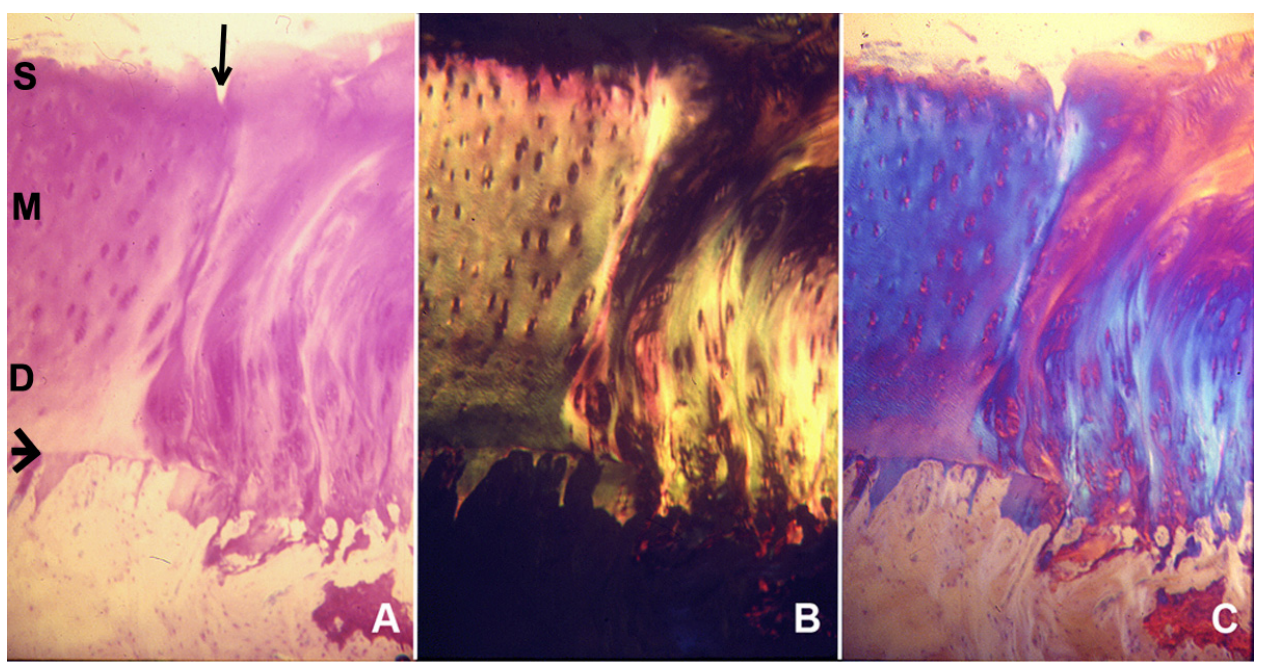

Fig. $6 A, B, C$. Articular cartilage section showing the host-graft interface region at 1 year after MP with a dimethylmethylene blue (DMMB) staining for proteoglycans (PGs) taken from the same visual field in normal (A) and polarised light microscope (B, C) without (B) and after (C) compensation of the optical anisotropy. The host-graft border is shown by a minor fissure of the articular surface (arrow). A partial transformation of hyaline cartilage to fibrocartilage in the graft alongside the host-graft interface can be observed as indicated by the irregular arrangement of chondrocytes (A) and the altered birefringence $(\mathrm{B}, \mathrm{C})$. The signs of altered birefringence: the extracellular matrix

(ECM) appears dark in polarised light (B), and unlike the blue anomalous polarisation colour generated by compensation in the extracellular matrix of the deep zone in the normally structured articular cartilage, an orange colour appears after compensation in a triangle-shaped area.

However, the majority of the ECM both in host and graft regions is characterised by highly oriented sulphated glycosaminoglycan side chains of PG molecules as shown by the intense birefringence (B). S: superficial zone, M: intermediate zone, D: deep zone, arrowhead: tide mark. Microscopic magnifications: $\times 20$

\section{Donor areas}

The decision to take 4-5 grafts always gave the surgeon a little flexibility in finding the most suitable graft for the drilled hole in the recipient area. In cases of non-perpendicular harvesting, five grafts were always harvested in this experiment.

The moderate effusion of the femoropatellar joint disappeared within two months after the first or second surgery. Other authors had similar results using the femoropatellar joint as a donor site (Tytherleigh-Strong et al., 2005). In the authors' experience, the described clinical picture makes it very unlikely that haemarthros was responsible for the mild effusion of the donor sites in this study.

Macroscopically, the donor areas appeared smooth and regular after 9 to 12 months (Fig. 3). In a younger animal hyaline cartilage had covered the entire surface of the previous canal. In older horses fibrocartilage was observed in the 2- to $3-\mathrm{mm}$ centre of the channels that were previously $6.5 \mathrm{~mm}$ in diameter. 
There is a remarkable difference in the repair capacity of weight-bearing and non-weight-bearing joint cartilage. In contrast to weight-bearing areas, hyalinelike cartilage formation was seen in non-weight-bearing areas four weeks after transplantation of perichondrial grafts in sheep (Bruns et al., 1992). The cranial surface of the MFT, which is a less weight-bearing joint surface, provided good circumstances for cartilage flow and acceptable quality of repair tissue with donor canals $6.5 \mathrm{~mm}$ in diameter in this study.

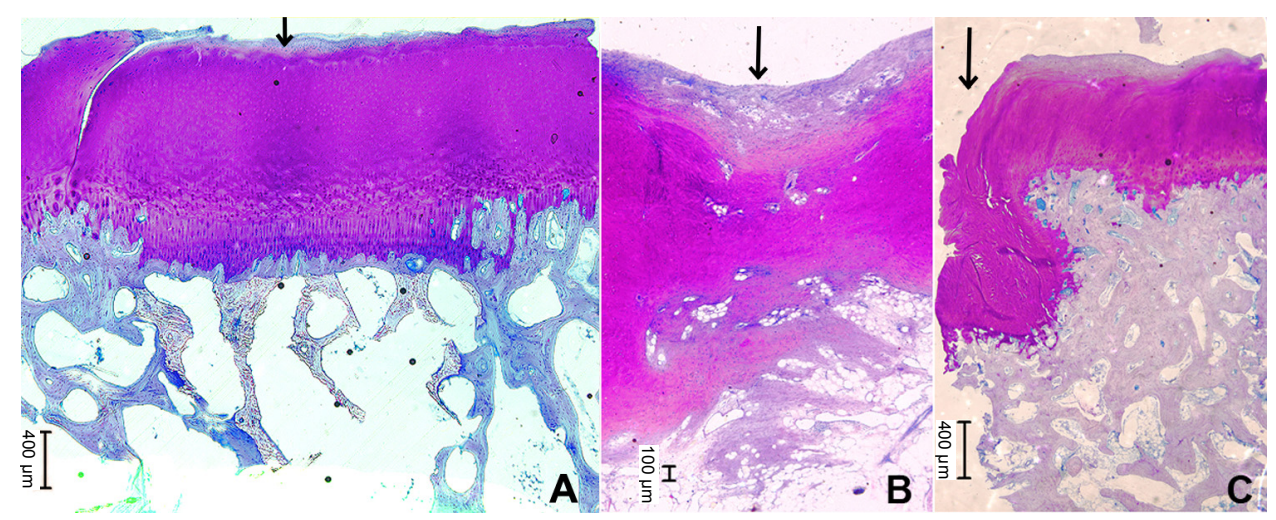

Fig. 7A, B, C. Articular cartilage sections showing tissue covering the donor canals 12 months after harvesting. A: high quality of hyaline cartilage covering the surface in a 20-month-old horse 12 months after harvesting. B: the centre of the donor canal (indicated by an arrow in pictures A, B,

C) 12 months after harvesting highlights a smooth gliding surface provided by fibrocartilage. $\mathrm{C}$ : one half of the donor canal 12 months following harvesting. The central part of this donor canal $6.5 \mathrm{~mm}$ in diameter was filled by fibrocartilage, while towards the periphery a hyaline-like cartilage could be observed, probably due to the phenomenon of cartilage flow from the marginal parts of the canal. The slides were stained with DMMB for proteoglycans (PGs)

\section{Recipient sites}

It is known that the ECM of normal articular cartilage is characterised by submicroscopically oriented collagen and GAG structure (Módis, 1991). A quantitative GAG evaluation and collagen typing was not performed in this study. However, examination of our histological sections under a polarised light microscope allowed us to evaluate the loss of GAG content as well as the maintenance or disappearance of the collagen arcades (Módis, 1991; Changoor et al., 2011) in the donor and recipient regions (Figs 5 and 6). In a large multicentre study with human mosaicplasty patients, good gliding surface and histologically proven survival of the transplanted hyaline cartilage was found in 81 of the 98 control arthroscopies (Hangody et al., 2008). In our study, around 7/10 biopsies showed similar results 12 months after transplantation despite immediate weight bearing.

Bony union has occurred at the subchondral level in most of the previously published MP studies involving different species (Hurtig et al., 2001; Pearce et al., 
2003; Huang et al., 2004; Harman et al., 2006; Cook et al., 2008). A tight seal in the gap between graft and normal cartilage (Tytherleigh-Strong et al., 2005) and rigid fixation of the transplant (Lane et al., 1977; Dew and Martin, 1992) seem to play an important role in preventing synovial fluid to penetrate into the subchondral area, possibly predisposing to the development of subchondral cysts.

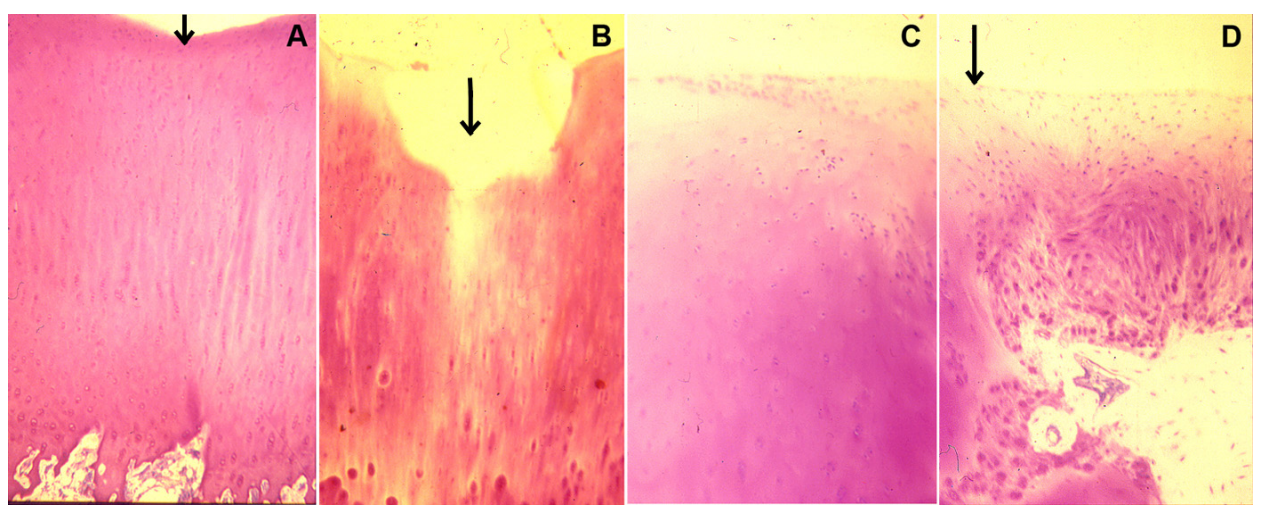

Fig. 8A, B, C, D. Articular cartilage sections showing the host-graft interface region after MP with DMMB staining for PGs. Samples were collected nine months (A, B), and one year (C, D) after the surgery. In Figs $8 \mathrm{~A}$ and $8 \mathrm{~B}$ the host-graft borders can only be recognised by moderate surface irregularities. Figure $8 \mathrm{C}$ demonstrates predominantly host region (with thicker articular cartilage), and Fig. 8D shows a predominantly graft area (with thinner cartilage). The cartilage matrix exhibits intense purple metachromasia typical of the normal hyaline cartilage except the superficial zones in $\mathrm{C}$ and $\mathrm{D}$. Regular arrangement of chondrocytes and chondrons are seen in A, B and C, suggesting a normal articular cartilage structure both in hosts and grafts. Chondrocytes are irregularly arranged in the graft, indicating full transformation into fibrocartilage, where the transplanted articular cartilage was significantly thinner than the surrounding articular cartilage (D).

$$
\text { Microscopic magnifications: } \times 20(\mathrm{~A}, \mathrm{~B}), \times 50(\mathrm{C}, \mathrm{D})
$$

In the authors' opinion, different cartilage thickness between transplanted and surrounding cartilage as well as no perpendicular insertion of some grafts was the reason for radiologically unevenly outlined subchondral bone in $4 / 10$ MFCs in this study, however, without any lytic or osteoarthritic changes visible on those radiographs.

If MP is used as a treatment for a smaller-sized lesion in human medicine, grafts are routinely implanted under arthroscopic control; however, with larger lesions a miniarthrotomic approach is preferred (Hangody et al., 2008). Arthroscopic transplantation needs a longer learning curve and can be definitively improved with more experience in the future in horses.

In a previous cadaver study it was stated that in horses kept in a paddock or at a lower activity level, the MP procedure is not advised above eleven years of age (Bodó et al., 2001). The reason for involving a 16-year-old horse in this in vivo study was to gain experience for further support of our cadaver study. 
This study has many limitations. The small number and the heterogeneous age of the horses were all limiting factors. It was not a terminal study, and therefore only one biopsy was taken per joint. Osteochondral biopsies were taken from distinctly visible interfaces only, decreasing the extrapolating informative value of those biopsies for the whole study. The horses had three weeks of hand walking after surgery, which was followed by turnout into small paddocks; however, the animals did not have controlled training exercise during the study. Clinical circumstances would have been modelled better with controlled exercise during the second half of the study.

We conclude that the horses were clinically sound three months after MP, and no signs of osteoarthritis could be detected 9-12 months postoperatively using radiography and follow-up arthroscopies. The histological results were good in young adults; however, in $50 \%$ of the samples examined, the transplanted hyaline cartilage showed GAG loss and transformation into fibrocartilage. The donor sites gave a satisfactory healing result clinically as well as histologically for this less weight-bearing surface.

Further experimental studies are required in the horse to clarify what other technical details need to be taken into account for achieving long-term histological survival of the transplanted hyaline cartilage in the equine MFC.

\section{Acknowledgements}

The authors would like to express their gratitude to Mrs Györgyné Nagy (Department of Anatomy, University of Debrecen) for preparing the histological sections. This study was supported by the Bolyai Foundation 2005-2008. The mosaicplasty instrument was provided by Metrimed Ltd., Hódmezővásárhely, Hungary.

\section{References}

Bodó, G., Hangody, L., Módis, L. and Hurtig, M. (2004): Autologous osteochondral grafting (mosaic arthroplasty) for treatment of subchondral cystic lesions in the equine stifle and fetlock joints. Vet. Surg. 33, 588-596.

Bodó, G., Hangody, L., Szabó, Zs., Peham, Ch., Schinzel, M., Girtler, D. and Sótonyi, P. (2000): Arthroscopic autologous osteochondral mosaicplasty for the treatment of subchondral cystic lesion in the medial femoral condyle in a horse. Acta Vet. Hung. 48, 343-354.

Bodó, G., Kaposi, A. D., Hangody, L., Tóth, J., Bakos, Z., Lukács, Z. and Péntek, G. (2001): The surgical technique as well as the age of the horse both influence the outcome of mosaicplasty in a cadaver equine stifle model. Acta Vet. Hung. 49, 111-116.

Bolam, C. J., Hurtig, M. B., Cruz, A. and McEwen, B. J. E. (2006): Characterization of experimentally induced post-traumatic osteoarthritis in the medial femorotibial joint of horses. Am. J. Vet. Res. 67, 433-447.

Bruns, J., Kersten, P., Lierse, W. and Silbermann, M. (1992): Autologous rib perichondrial grafts in experimentally induced osteochondral lesions in the sheep knee joint: morphological results. Virchows Arch. A Pathol. Anat. Histopathol. 421, 1-8. 
Changoor, A., Tran-Khanh, N., Méthot, S., Garon, M., Hurtig, M. B., Shive, M. S. and Buschmann, M. D. (2011): A polarized light microscopy method for accurate and reliable grading of collagen organization in cartilage repair. Osteoarthr. Cartilage 19, 126-135.

Constantine, V. S. and Mowry, R. W. (1968): The selective staining of human dermal collagen. II. The use of picrosirius red F3B with polarization microscopy. J. Invest. Dermatol. 50, 419-423.

Cook, J. L., Hudson, C. C. and Kuroki, K. (2008): Autogenous osteochondral grafting for treatment of stifle osteochondrosis in dogs. Vet. Surg. 37, 311-321.

Dew, T. L. and Martin, R. A. (1992): Functional, radiographic, and histologic assessment of healing of autogenous osteochondral grafts and full-thickness cartilage defects in the talus of dogs. Am. J. Vet. Res. 53, 2141-2152.

Frisbie, D. D., Trotter, G. W., Powers, B. E., Rodkey, W. G., Stedman, J. R., Howard, R. D., Park, R. D. and McIlwraith, C. W. (1999): Arthroscopic subchondral bone plate microfracture technique augments healing of large chondral defects in the radial carpal bone and medial femoral condyle of horses. Vet. Surg. 28, 242-255.

Hangody, L., Dobos, J., Balo, E., Panics, G., Hangody, R. L. and Berkes, I. (2010): Clinical experiences with autologous osteochondral mosaicplasty in an athletic population. Am. J. Sports Med. 38, 1125-1133.

Hangody, L., Kish, G., Kárpáti, Z., Szerb, I., Udvarhelyi, I., Toth, J., Dioszegi, Z. and Kendik, Zs. (1997): Autogenous osteochondral graft technique for replacing knee cartilage defects in dogs. Orthopedics International Edition 5, 175-181.

Hangody, L., Vásárhelyi, G., Hangody, L. R., Sükösd, Z., Tibay, Gy., Bartha, L. and Bodó, G. (2008): Autologous osteochondral grafting - technique and long-term results. Injury 39, 32-39.

Harman, B. D., Weeden, S. H., Lichota, D. K. and Brindley, G. W. (2006): Osteochondral autograft transplantation in the porcine knee. Am. J. Sports Med. 34, 913-918.

Huang, F. S., Simonian, P. T., Norman, A. G. and Clark, J. M. (2004): Effects of small incongruities in a sheep model of osteochondral autografting. Am. J. Sports Med. 32, 1842-1848.

Hurtig, M., Pearce, S., Warren, S., Kalra, M. and Miniaci, A. (2001): Arthroscopic mosaic arthroplasty in the equine third carpal bone. Vet. Surg. 30, 228-239.

Jackson, W. A., Stick, J. A., Arnoczky, S. P. and Nickels, F. A. (2000): The effect of compacted cancellous bone grafting on the healing of subchondral bone defects of the medial femoral condyle in horses. Vet. Surg. 29, 8-16.

Janicek, J. C., Cook, J. L., Wilson, D. A. and Ketzner, K. M. (2010): Multiple osteochondral autografts for treatment of a medial trochlear ridge subchondral cystic lesion in the equine tarsus. Vet. Surg. 39, 95-100.

Lane, J. M., Brighton, C. T., Ottens, H. R. and Lipton, M. (1977): Joint resurfacing in the rabbit using an autologous osteochondral graft. J. Bone Joint Surg. Am. 59, 218-222.

Martinek, V., Ueblacker, P. and Imhoff, A. B. (2003): Current concepts of gene therapy and cartilage repair. Br. Ed. J. Bone Joint Surg. 85, 782-788.

Módis, L. (1991): Organization of the Extracellular Matrix: A Polarization Microscopic Approach. CRC Press, Boca Ration, Florida.

Nixon, J. A., Begum, L., Mohammed, O. H., Huibregtse, B., O'Callaghan, M. M. and Matthews, G. L. (2011): Autologous chondrocyte implantation drives early chondrogenesis and organized repair in extensive full- and partial-thickness cartilage defects in an equine model. J. Orthop. Res. 29, 1121-1130.

Ortved, K. F., Nixon, A. J., Mohammed, H. O. and Fortier, L. A. (2012): Treatment of subchondral cystic lesions of the medial femoral condyle of mature horses with growth factor enhanced chondrocyte grafts: a retrospective study of 49 cases. Equine Vet. J. 44, 606-613.

Pearce, S. G., Hurtig, M. B., Boure, L. P., Radcliffe, R. M. and Richardson, D. W. (2003): Cylindrical press-fit osteochondral allografts for resurfacing the equine metatarsophalangeal joint. Vet. Surg. 32, 220-230.

Tytherleigh-Strong, G., Hurtig, M. and Miniaci, A. (2005): Intra-articular hyaluronan following autogenous osteochondral grafting of the knee. Arthroscopy 21, 999-1005. 